\title{
A case of primary vulva epidermoid cyst
}

Chu Liu ${ }^{1}$, Xiaohui Guo ${ }^{1}$, Hua Lan ${ }^{1}$, Da Zeng ${ }^{1}$, Jing Yuan ${ }^{1}$, Jiahui Yong ${ }^{1}$, and Songshu $\mathrm{Xiao}^{1}$

${ }^{1}$ Central South University Third Xiangya Hospital

June 7, 2021

\begin{abstract}
In this report, We discuss a case of epidermoid cyst originating from the female vulva, which was completely excised by surgery. Pathologically confirmed as a vulva epidermoid cyst.
\end{abstract}

Case Report

A case of primary vulva epidermoid cyst

Chu Liu ${ }^{\mathrm{a}}$, Xiaohui Guo ${ }^{\mathrm{a}}$, Hua Lan ${ }^{\mathrm{a}}$, Da Zenga ${ }^{\mathrm{a}}$, Jing Yuan ${ }^{\mathrm{a}}$, JiaHui Yong $^{\mathrm{a}}$, Songshu Xiao ${ }^{\text {** }}$

${ }^{a}$ Department of Gynecology and Obstetrics, The Third Xiangya Hospital, Central South University, Changsha, Hunan, China

Corresponding Author:

Songshu Xiao

Department of Gynecology and Obstetrics, the third Xiangya Hospital, Central South University

Changsha, Hunan, 410013, People's Republic of China.

Tel: 13787412954

Email: xiaosongshu@csu.edu.cn

Number of Figures: 3

Word count: 834

Keywords: vulva, perineum, Epidermoid cyst

Established Facts

Already known fact that primary or secondary epidermoid cysts occurring in female external genitals are more common in the clitoris.

Novel Insights

New information shows in this case report, Epidermoid cysts occurring in the vulva. And the First diagnosis is vestibular gland cyst. Through this case report, the clinician can raise awareness of differential diagnosis between primary vulva Epidermoid cysts and vestibular gland cyst. 


\begin{abstract}
Introduction: Epidermoid cyst is a benign tumor, which is one of the most common skin tumors. It usually occurs in the maxillofacial,occasionally in the sublingual, intracranial and spleen, etc. But rare epidermoid cyst originating from the female vulva.Case presentation: In this report, We discuss a case of epidermoid cyst originating from the female vulva, which was completely excised by surgery. Pathologically confirmed as a vulva epidermoid cyst.Conclusions: It has been reported that primary or secondary epidermoid cysts occurring in female external genitals are more common in the clitoris, and the clinical manifestation is clitoris hypertrophy. The site of epidermoid cysts in this case is rare and it is easy to be misdiagnosed as vestibular gland cyst. Therefore, the clinician should strengthen the differential diagnosis.
\end{abstract}

Introduction: Epidermoid cyst is a benign tumor, which is one of the most common skin tumors, occasionally occurring under the tongue, intracranial and spleen. Primary epidermoid cysts may be derived from ectodermal vestigial tissue ${ }^{1}$. The clinical manifestation of primary vulva epidermoid cysts is painless vulva mass. If the cyst increases further, it may affect walking. Local color doppler ultrasound and nuclear magnetic resonance can assist diagnosis, and pathological results are the gold standard for diagnosis. The main treatment is surgical excision of cyst.

Case presentation: A 29-year-old women consulted for a vulva mass discovered at the age of 27. The patient first noticed this mass when it was very tiny while two years ago, about the size of a mung bean, and then it started growing over the last 2 years. Without pain or discomfort of walking. Perineal examination revealed a $3 \mathrm{~cm} \times 3 \mathrm{~cm}$, smooth surface, normal skin temperature, clear boundary and no tenderness cystic swelling between below the right labia majora and outboard the perineal body (Figure 1). Vaginal patency, a small amount of white odor-free secretions. Smooth cervix, normal size, medium texture. No obvious abnormalities were observed in bilateral attachments. No palpable enlarged lymph nodes in the groin. Local color doppler ultrasound of vulva: A $27 \mathrm{~mm} \times 16 \mathrm{~mm}$ unechoic region with clear boundary can be consideration it may be a vestibular gland cyst. Blood routine and coagulation function were normal. Preliminary diagnosis of vestibular gland cyst. Immediately, the patient was scheduled for surgery, the mass of vulva was removed under the venous general anesthesia, The pedicle of this cyst was found to be tightly adhering to the skin, and the mass was stripped completely(Figure 2), after that, we performed an effective hemostasis and the incision was primarily closed with no-tension simple stitches. The gross specimen shows that there are yellow-white bean dregs and sebum inside in the cyst, and no nipples or hair in it. The cyst wall is smooth. Postoperative pathologic examination (Figure 3)show that benign cyst with layered squamous epithelium lining the cyst wall, containing multiple keratinites. Pathology confirmed that it is a vulva epidermoid cyst.

Discussion: Epidermoid cysts tend to occur on the face, head and neck, the wall of the cyst is thin. Histopathological appearance of the epidermoid cyst lined by stratified squamous epithelium and filled with keratinous material, The latter is also the most important point to differentiate from dermatoid cysts ${ }^{2}$. In the case of epidermoid cysts occurring in female genitalia, more located in the clitoris were reported, including primary epidermoid cysts in the clitoris and Secondary clitoris epidermoid cysts. Primary clitoris epidermoid cysts may be accompanied by sex deformity ${ }^{3}$, clinical manifestations are non-hormonal clitoris hypertrophy; The formation of a secondary epidermoid cyst can be caused by trauma or surgery leading to the implantation of the epidermis into the dermis, such as clitoris epidermoid cysts formed after female genital mutilation ${ }^{4-6}$, common in sub-Saharan Africa. In addition, Huang Jing et al reported a case of vaginal epidermoid cyst $^{1}$. There are few epidermoid cysts located between the lower labia majora and the perineal body. The vulvar epidermoid cysts are often misdiagnosed as vestibular gland cysts. Because epidermoid cysts may be malignant, the first choice for treatment is surgery. It is difficult to distinguish vulva epidermoid cyst from vestibular gland cyst before operation, so in the case of undiagnosed, we should pay more attention to the separation of cyst wall, avoid the rupture of cyst during the operation, form an iatrogenic epidermoid cyst. On the other hand, the cyst should be stripped as clean as possible, if the cyst wall remains, it may lead to recurrence or even malignant change in the future ${ }^{7}$. Due to the abundant blood supply in the vulva, attention should be paid to hemostasis during operation to avoid the formation of subcutaneous hematoma.

Conclusions: It has been reported that primary or secondary epidermoid cysts occurring in female external 
genitals are more common in the clitoris. Epidermoid cysts occurring in the vulva is easy to be misdiagnosed as vestibular gland cyst. But epidermoid cysts may be malignant. So, it is our hope that through this case report, the clinician can raise awareness of differential diagnosis between primary vulva Epidermoid cysts and vestibular gland cyst.

\section{Acknowledgement}

We thank the patient and her family for allowing us to share her details, and we thank all the staff of The Third Xiangya Hospital.

\section{Ethics approval and consent to participate}

Not applicable.

\section{Consent for publication}

Written informed consent was obtained from the patient for publication of this case report and any accompanying images. A copy of the written consent is available for review by the Editor-in-Chief of this journal.

\section{Competing interests}

The authors declare that they have no competing interests.

\section{Availability of data and materials}

The authors agree to make the raw data and materials described in this report freely available.

\section{Funding}

The project of hunan provincial natural science foundation(2018JJ3782)

\section{Authors' contributions}

LC is the first authors. LC, GXH, LH, YJ, ZD and YJH assembled, analyzed, and interpreted the patient data. All authors contributed to writing the manuscript. All authors read and approved the final manuscript.

\section{Reference}

1. Jing Huang, Junfeng Kong, jiebin Yang, et al. A case of vaginal epidermoid cyst. J Journal of Practical Radiology.2018,34(12):1990-1991.DOI:10.3969/j.issn.1002-1671.2018.12.051.

2. Tonghua Liu, Jin Chen, et al. Liu Tonghua Diagnostic Pathology[M].Beijing:People's Medical Publishing House, 2018:37.

3. Aggarwal SK, Manchanda V, Pant N. Epidermoid cyst of clitoris mimicking clitoromegaly. J Indian Assoc Pediatr Surg. 2010;15(1):23-24. doi:10.4103/0971-9261.69137.

4. Birge O, Erkan MM, Serin AN. Case report: epidermoid inclusion cyst of the clitoris as a long-term complication of female genital mutilation. J Med Case Rep. 2019;13(1):109. Published 2019 Apr 27. doi:10.1186/s13256-019-2035-6.

5. Anderson-Mueller BE, Laudenschlager MD, Hansen KA. Epidermoid cyst of the clitoris: an unusual cause of clitoromegaly in a patient without history of previous female circumcision. J Pediatr Adolesc Gynecol. 2009;22(5):e130-e132. doi:10.1016/j.jpag.2008.10.006.

6. Ofodile FA, Oluwasanmi JO. Post-circumcision epidermoid inclusion cysts of the clitoris. Plast Reconstr Surg. 1979;63(4):485-486. doi:10.1097/00006534-197904000-00007.

7. Bijun Tan, Lixia Chen. A case of ultrasound findings of carcinogenesis of vulvar epidermoid cysts. J Chinese Journal of Ultrasound in Medicine.2020,36(2):174.

\section{Figure Legends}




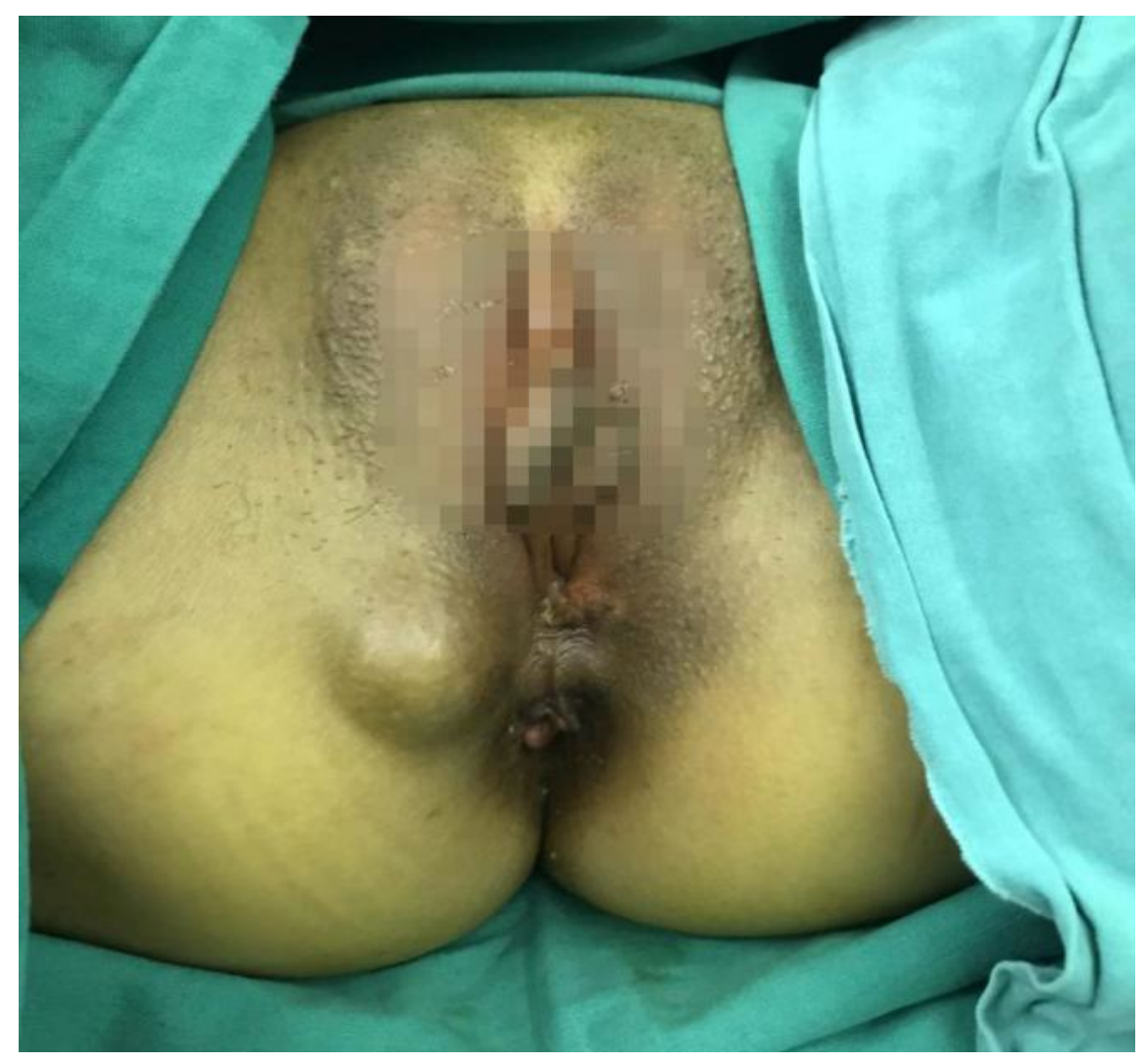

Fig. 1. Preoperative inspection of vulvar region 


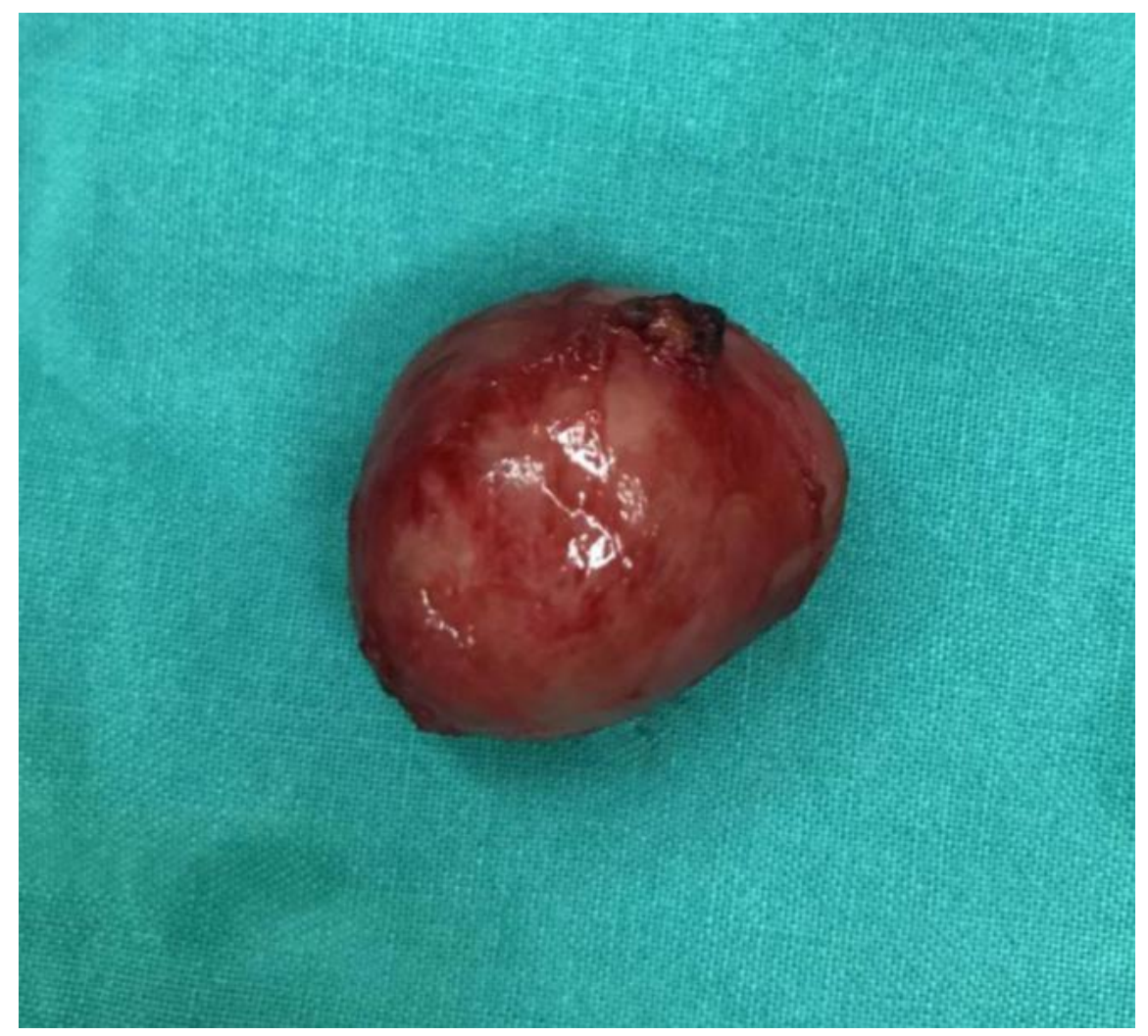

Fig. 2. Intraoperative appearance of the cyst

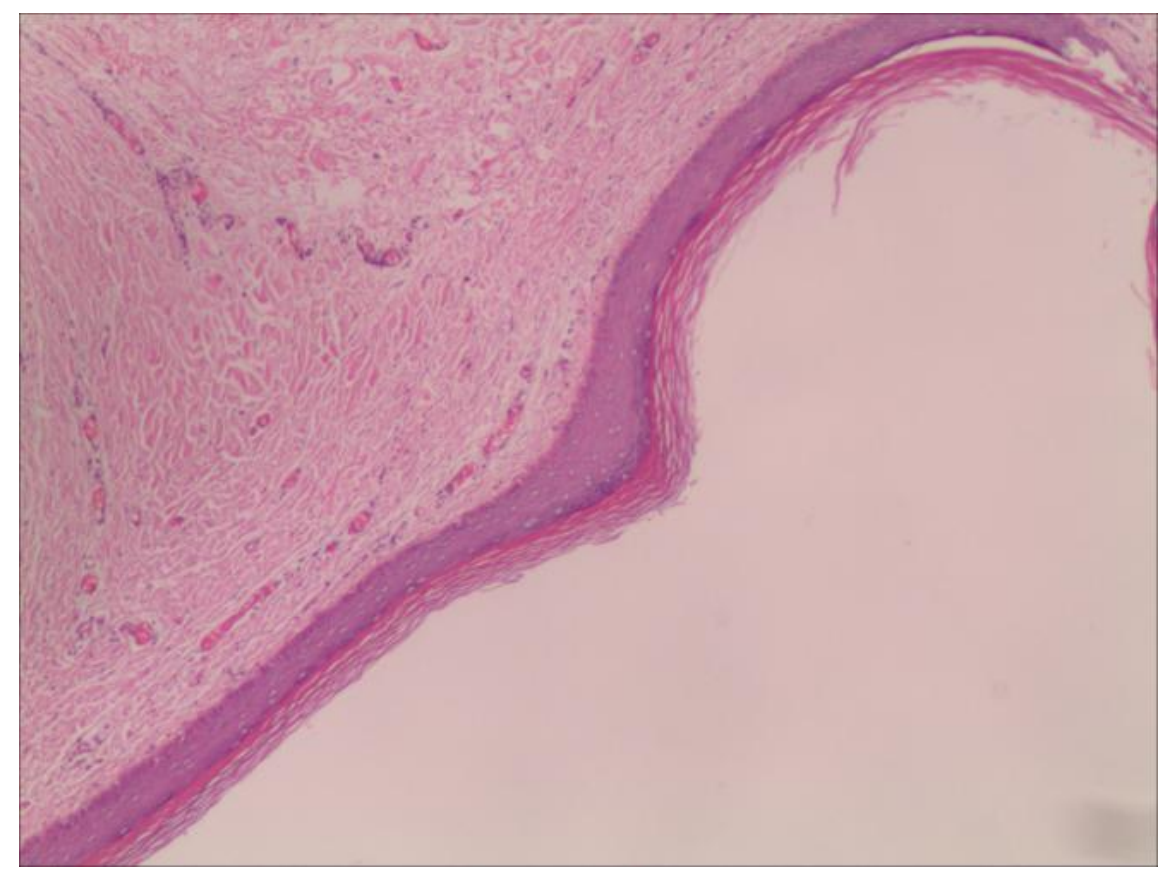

Fig.3. Histopathological appearance of the vulva epidermoid cyst(H\&E stain, magnification $\times 200)$ 


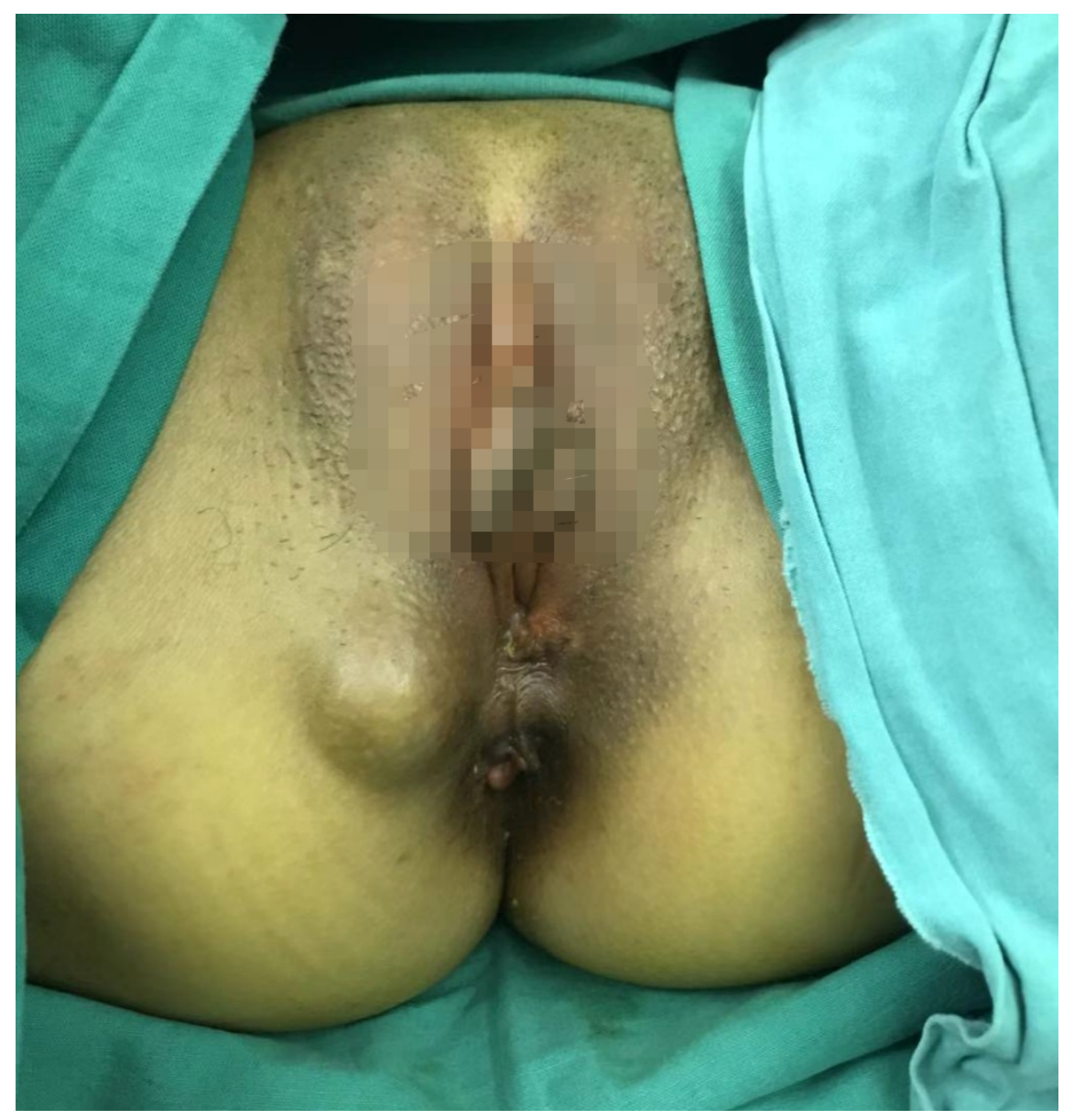



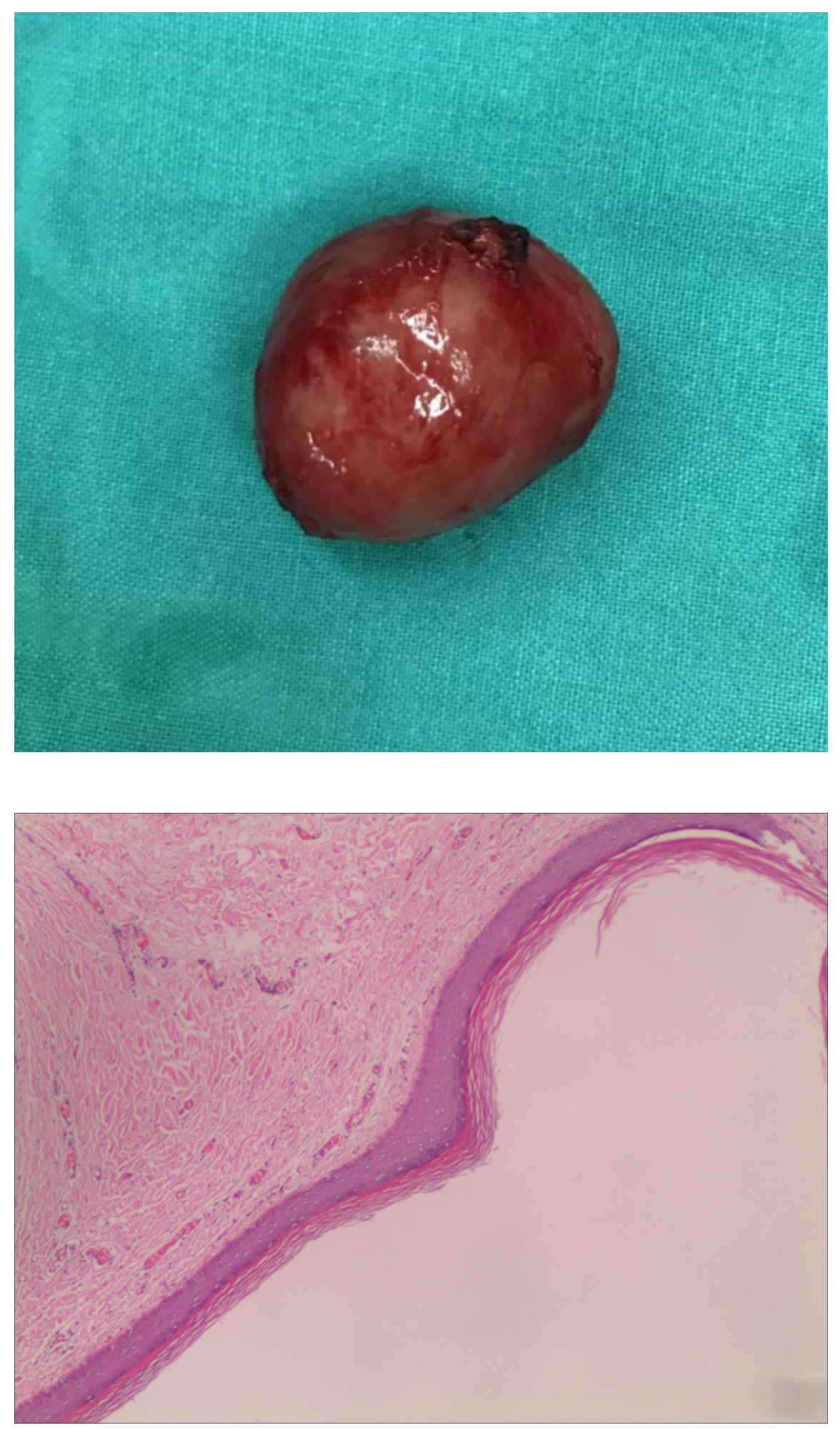